\title{
Accelerator Science and Technology in Europe EuCARD 2012
}

\author{
Ryszard S. Romaniuk
}

\begin{abstract}
Accelerator science and technology is one of a key enablers of the developments in the particle physics, photon physics, electronics and photonics, also applications in medicine and industry. The paper presents a digest of the research results in accelerators in Europe, shown during the third annual meeting of the EuCARD - European Coordination of Accelerator Research and Development. EuCARD concerns building of research infrastructure, including advanced photonic and electronic systems for servicing large high energy physics experiments. There are debated a few basic groups of such systems like: measurement - control networks of large extent, multichannel systems for metrological data acquisition, precision photonic networks for reference time distribution.
\end{abstract}

Keywords-Electronics and photonics for high energy physics experiments, free electron laser, advanced electronic systems, integration of hardware and software, nuclear electronics.

\section{INTRODUCTION}

T HE EUCARD 2012 ANNUAL CONFERENCE on the development of the accelerator research infrastructure in Europe was held in Warsaw on 24-27 April. The venue of the meeting was the Warsaw University of Technology, Faculty of Electronics and Information Technologies. Around 100 accelerator researchers participated and 60 invited papers were presented. The conference covered the following subjects: new materials for building accelerators, research infrastructure for particle measurements, muon electronics, upgrade of existing accelerators, HL-LHC, HE-LHC, new infrastructure of large scale, experiments of new physics, plasma wake field and laser accelerators. The aim of the conference was to summarize annual achievements of the European FP7 project co-financed by the EU [1]-[60].

\section{European AcCelerator Network - ACCNet}

ACCNET is an European Accelerator Science Network, an activity within the EuCARD project, with coordination and management duties, run by representatives of the following institutions, which possess large accelerator infrastructure IN2P3 and CNRS-Paris, GSI-Darmstadt, CERN-Geneva, and DESYHamburg. ACCNET consists of three activities/networks: EUROLUMI - accelerator and colliders performance; RFTECH - superconducting and normal conducting RF technologies; EuroNNAC - European Network for Novel Accelerators.

EUROLUMI brings together experts in beam dynamics, magnets, collimation, RF and experiments. EUROLUMI is involved in: FAIR (facility for antiproton and ion research);

Ryszard S. Romaniuk is with Warsaw University of Technology, Institute of Electronic Systems, Nowowiejska 15/19, Warsaw, Poland (e-mail: rrom@ise.pw.edu.pl).
LHC IR (large hadron collider, interaction region) and beam parameter upgrade (ES - early separation, FCC - full crab crossing, LE - low emittance, LPA - large Piwinski angle); CERN complex upgrade; LHC injector upgrade; initial initiative on plasma acceleration.

RFTECH brings together RF experts from different labs, proton and electron accelerators, like CLIC (compact linear collider) and ILC (international linear collider), FLASH, E-XFEL, GANIL, SPIRAL2, SPL, LHC, Neutrinos. Encompasses all aspects of RF technology e.g. klystron development, RF power distribution system, cavity design, and low-level RF system, for linear accelerators, storage rings, and associated research infrastructures, including transversely deflecting (crab) cavities and financial aspects such as costing tools.

EuroNNAc identifies possible usage of advanced accelerator techniques in large scale conventional facilities and a roadmap towards an advanced accelerator facility for big science with applications in applied and fundamental research. EuroNNAC proposes new European facilities, identifies synergies, defines roadmaps, builds bridges, between plasma science, laser science, ultra-fast science and accelerator science.

The ACCNet tools are: publications, annual workshop, topical meetings and mini-workshops, capability of inviting and/or exchange experts over periods of typically a week to a month, exploratory studies and collaborations, opportunities for students, unique place of discussion with users. The exchange of ideas and expertise are aiming at identifying the most promising strategies and technologies. AccNet is interacting with other related EU initiatives like LHC High Luminosity, ILC Preparatory Phase. AccNet is a catalyzer between LHC experiments, US Programs and US Labs US-LARP, APL, ITER, E-XFEL, ESS (European Spallation Source), FAIR, HEP and accelerator lab Users, Japanese Labs - KEK, J-PARC, Russian Labs, European Labs and Universities, other pan-European research ventures, ESA, South American Labs in Brazil and Mexico - CINVESTAV, etc. AccNet operates via its web site http://cern.ch/accnet.

During the last year AccNet organized and co-organized over a dozen of conferences, workshops and meetings. Advanced Techniques in LLRF for XFEL, Cracow, April 2012, covered the topics: towards CW operation of XFEL laser, summary of 9mA operation of FLASH laser, development of $\mu$ TCA based LLRF system, RF synchronization system for XFEL, LLRF software development for XFEL. The goal of $1^{\text {st }}$ EuroNNAc general workshop, CERN May 2011, was to form the network and define the work towards a significant FP8 proposal for novel accelerator facilities in Europe. RFTech meeting, Gliwice, June 2011, was devoted to application of 
xTCA standards for instrumentation in LLRF FLASH and XFEL in DESY and CODAC in ITER, vector modulators, low phase noise PLL for femtosecond precision RF field detection, uTCA based controllers and associated software. OMCM Optics Measurements, Corrections and Modeling for HighPerformance Storage Rings, CERN, June 2011, was debated the following areas: motivation of HE machines, colliders, HI machines, light sources and damping rings, experience from colliders, high energy and intensity machines, beam diagnostics. MulCoPim, Valencia, Spetember 2011, topics were: multipactor components, multipactor Tx lines, multipactor dielectrics, corona, multipactor experimental, software, multicarrier, PIM, SEY and accelerators. LLRF Workshop, DESY, October 2011, had the following sessions: reviews of the lowlevel RF system at various laboratories, fast and/or digital lowlevel control for pulsed and $\mathrm{CW}$ operation, applications of the digital signal processing at accelerators, software development, LLRF systems - requirements, performance reached, modeling. The $5^{\text {th }}$ Workshop on LHC crab cavities, CERN, November 2011, debated: convergence to 2 or 3 compact crab cavity design, first $\mathrm{Al}$ prototypes, detailed cavity specifications relevant to HL-LHC parameters, beam tests of the compact design in the SPS and the LHC. The $3^{\text {rd }}$ annual RFTech meeting, Rostock, December 2011, topics were: electromagnetic field computation by finite integration, finite elements method and perturbative computation; cavity RF models - coupled multicell cavities with beam, cavity model and experiment validation for gradient flatness procedure; low-level RF and regulation. General topics were: RF panorama at CERN, activities at Darmstadat GSI and GANIL - Spiral2, and crab cavities. LLRF Collaboration Meeting, Warsaw, December 2011, the main topics were: integration of hardware and software, solutions for the LLRF and synchronization, systems at FLASH and XFEL.

EuroLumi gives input to LHC upgrade, strategy for LHC crab cavities, HL-LHC parameters, LPA scenario, flat beam crab waist scenario, e-cloud mitigation, HL-LHC e-cloud, revisions of LHC upgrade plans, energy upgrade. RFTech organizes the RF community. EuroNNAc organizes novel accelerator community. In summary (F. Zimmerman, CERN), AccNet is breaking new grounds in plasma acceleration, crystal collimation, new contacts with ESA, CINVESTAV, VALSPACE, MIT, Kyoto Uni., and Hiroshima Uni.

\section{CRAB CAVities FOR High Luminosity LHC} (HL-LHC)

Increasing the luminosity in HL-LHC upgrade results in smaller $\mathrm{B}^{*}$, increase the long range effect at the interaction region (IR), thus, sufficiently large crossing angle is inevitable. Large crossing angle reduces the long range effect but also reduces luminosity via decreased geometric overlap of bunches. The bunches have to be skewed to increase the efficiency of particle collisions during the bunch crossing (BX). Rotating the bunches with crab cavities (CC) before and after collision can reduce the effect of larger crossing angle (G.Burt, Lancaster Uni.). The aim of HL-LHC project, via the injector, linacs, booster, SPS and the IR upgrades is to obtain $2 \times 10^{35} \mathrm{~cm}^{-2} \mathrm{~s}^{-1}$ in around 2022 . The main difficulty is that crab cavities have to be really compact due to the limited space, close to the IR, where the opposing beamlines are in very proximity to each other. In practice, the CC must fit within $143 \mathrm{~mm}$ radius. Using $800 \mathrm{MHz}$ RF causes a S-shaped bunch which reduces luminosity, hence a $400 \mathrm{MHz}$ compact cavity is desired.

Four years of design evolution results in several basic models, of which three are leading: $\lambda / 4$ TEM cavity by BNL, $\lambda / 2$ TEM cavity (double ridge) by ODU and SLAC, 4 Rod crab cavity by Cockroft and JLab. The $\lambda / 4$ TEM cavity is very short in the direction of opposing beamline. The nearest HOM (higher order mode) is far away. It has a pedestal inside to cancel $\mathrm{E}_{\mathrm{z}}$ field component. Using the $\lambda / 2$ TEM cavity removes any monopole and quadrupole components. However, it then is only compact in one direction, while LHC may need both planes. Also it has another monopole mode nearby. The $4 \mathrm{R}$ cavity is ultra compact as it has its half wavelength in the longitudinal plane. CEBAF has a normal conducting version as a separator. It has a lower order mode but less HOMs. For the cavity to be useful the following parameters/processes have to be assessed, simulated and the measured: multipactor simulations, HOM damping, and RF field nonlinearity. Multipactor, e-cloud generation and multiplication from cavity wall edges and ridges, has been modeled in all three cavities. Although multipactor is found, it disappears for clean surfaces suggesting it can be processed through. The HOMs and LOMs have to be damped, due to their location in LHC. The lowest monopole mode will need quality factor 100 and may have up to several $\mathrm{kW}$ in the HOM/LOM coupler. Each cavity, out of the three mentioned, has its own set of couplers, although each set probably works for all cavities. The EM fields in complex shapes cavities are not pure dipole. Large sextupole components are also existing. Altering the shape of the rods in each of the cavities can reduce the sextupole components. Niobium prototypes of the cavities were built and very initial tests done at CERN. Crab cavities cannot be tested at LHC. COLDEX location in the SPS (super proton synchrotron) has a bypass line that could serve as a hadron crab cavity test location, prior to LHC. The goals of SPS tests, predicted in 2015, are: cavity validation with beam including field ramping, RF controls and impedance; collimation, machine protection, cavity transparency, RF noise, emittance growth, non-linearities; instrumentation and interlocks.

\section{NORMAL CONDUCTING LinACS}

In parallel to works on the SC linac (ILC) the research is continued on CLIC (compact linear collider) with the CTF3 experiment (CLIC test facility) (E. Jensen, CERN, G. Blair, RHUL). CLIC is expected to reach multi $\mathrm{TeV}, 100 \mathrm{MV} / \mathrm{m}$, $12 \mathrm{GHz}$ beams with the length of nearly $50 \mathrm{~km}$ and power supply in a form of a $15 \mathrm{MW}, 1 \mathrm{GHz}$ drive beam. CLEX facility is developed, with a two-beam module. The studies are on high precision assembly and breakdown studies, HOM damping, diagnostic equipment, PETS design and application. CLEX works with 100A drive beam and 1A main beam. PETS were designed and fabricated by CIEMAT. Coupler 
machining was completed and couplers were successfully tested. HOM damping studies are done at UNIMAN using detuned damping structure DDSA. Machining of a complete accelerating structure is under way, using prototype disks. New hybrid structure largely improves the performances of conventional DDS but further studies are needed. Breakdown studies are done by the UH. Multi-scale model is developed to understand the mechanisms in or close to the surface of the materials due to the effect of static electric field. The multiscale model is used for: simulating plastic deformations of metal surfaces due to tensile stresses leading to tips on the surface and internal single voids; combining electro-dynamic effects and atomistic simulations to predict behavior of surface atoms; simulation of created plasma and subsequent surface damage. A single void can also act as a source, and was shown that this can lead to major growth on top of the void, as one of the origins of tips. High precision assembly is of concern. It requires development of tooling for the assembly of the accelerating structures and PETS, as well as thermomechanical simulation of the entire assembled structures.

Diagnostic equipment includes: surface quality measurements, new BPM (beam position monitoring), new MTV and FLASH box, phase monitors, cavity BPM long term stability. The average resolution of BPM system was 200nm, with the best resolution $27 \mathrm{~nm}$ (RHUL). The interaction point (IP) region requires special attention. 4BPMs are to monitor the beam focus. Calibration is difficult due to large orbit changes. Calibration improves with jitter subtraction. Two calibration constants are required for each BPM - amplitude and phase. The CLIC crab-cavity induces a higher order correlation in the beam dynamics at the IP, leading to luminosity loss within the reference CLIC BDS design. To compensate for this effect, simulation work was performed on re-optimizing the nonlinear elements in the CLIC BDS, between the crab-cavity and the IP, to minimize the luminosity loss and restore the design luminosity of the machine. This fact highlights also the need for nonlinear optimizations of other areas of the IP vicinity. A separate issue is precision alignment and nanometer scale stabilization of the CLIC IP quadrupole magnets (A. Jeremie, CERN). Ground motion has an impact on the luminosity, especially when the beam guiding quadrupole magnets vibrate. The aim parameters for focus stability at the IP with mechanical and beam feedback are $0.1 \mathrm{~nm}$ at $0.1 \mathrm{~Hz}$.

\section{Advanced Materials for Next Generation COLlimators}

Particle beam propagation is associated with unavoidable losses. The losses include: primary halo of protons - managed by primary collimator, secondary halo - managed by secondary collimator and tertiary halo managed by absorber. At each stage there are $e$ and $\pi$ showers accompanying $\mathrm{p}$ halos. Behind the absorber (made of $\mathrm{W}$ ) there are SC magnets and particle physics experiments. Simulations and beam measurements on beam halo are researched and aimed to improve the performance of the actual LHC collimation system and to support its upgrade (J. Stadlmann, GSI Darmstadt). Some of the many research topic include: combined betatron and momentum cleaning, misalignment studies and nonlinear collimation. In some solutions, like in SIS 100, the primary collimator is a thin foil which acts like a scatterer of the halo particles and the secondary collimators in a form of bulky blocks are necessary to absorb the scattered particles. Beam trajectories are calculated using MAD-X, MERLIN, SixTrack and FLUKA. These studies will also improve the BPM (Beam Position Measurement) and control system as well as collimation efficiency. Crystals (acting as primary aperture) assisted collimation assumes usage of elastic and diffractive scattering. The crystal is bent and behaves like a Bragg grating for scattered particles.

The collimation system must satisfy two main functions: Multi-stage Beam Cleaning (BC), i.e. removing stray particles which would induce quenches in SC magnets; and Machine Protection, i.e. shielding the other machine components from the catastrophic consequences of beam orbit errors. Classical C-C Coal collimators are affected by intrinsic limitations which may ultimately limit LHC performances: low Z-material of the collimator limits cleaning efficiency, poor electrical conductivity material of collimator means high RF impedance, limited radiation hardness of collimator material means reduced lifetime. Innovative materials are needed for accelerator collimator jaws for the upgrade of the LHC (A. Bertarelli, CERN). The research on collimator materials ColMat consists of R\&D of novel materials, advanced numerical simulations, material testing, prototype design and manufacturing. ColMat R\&D focuses on Metal Matrix Composites (MMC) with diamond and graphite reinforcement as they have the potential to combine the properties of diamond and graphite (high $\mathrm{k}$, low rho, low CTE) with those of metals (strength, high Poisson ratio, high Young's modulus). Sintering techniques include rapid hot pressing (RHP) and liquid infiltration. Spark plasma sintering (SPS) is a technology of the future. Materials under investigation are Copper-diamond (Cu-CD), Molybdenumdiamond (Mo-CD), Silver-diamond (Ag-CD), MolybdenumGraphite (Mo-Gr). The materials are tested for: shock wave analysis, smooth particle hydrodynamics (material fragmentation), irradiation studies with proton and carbon ion beams swelling measurements, mechanical tests.

$\mathrm{Cu}-\mathrm{CD}$ composites are produced by RHP from $60 \%$ diamond and $40 \% \mathrm{Cu}$. No diamond degradation in reducing atmosphere is observed. Diamond graphitization starts at approximately $1300^{\circ} \mathrm{C}$. Thermal conductivity is around $500 \mathrm{~W} / \mathrm{mK}$, and electrical conductivity $12.6 \mathrm{MS} / \mathrm{m}$. There is no direct interface between $\mathrm{Cu}$ and $\mathrm{CD}$ because of a lack of affinity. Mechanical strength is average $120 \mathrm{MPa}$. Material is brittle. $\mathrm{Cu}$ low melting point limits applications for highly energetic accidents.

Ag-CD composite consists in $60 \%$ with diamond and $40 \%$ with Ag-Si alloy. There is an excellent bonding between $\mathrm{Ag}$ and $\mathrm{CD}$ assured by $\mathrm{SiC}$ formation on diamond. The material features high mechanical flexural strength 500MPa, high electrical conductivity, low melting of Ag-Si eutectic phase $840^{\circ} \mathrm{C}$, and low homogeneity, the latter preventing manufacturing of large components.

Mo-CD composites. High sintering temperature of Mo $\left(1700^{\circ} \mathrm{C}\right)$ leads to diamond graphitization. The solution is 
to apply LPS (liquid phase sintering) or ASS (solid-state sintering). LPS is based on addition of low-melting phase to fill the pores between Mo and CD. ASS is based on addition of activating elements like $\mathrm{Ni}, \mathrm{Pd}$ which enhance Mo sintering at low temperature around $1300^{\circ} \mathrm{C}$.

Mo-Gr composites are under intense R\&D. Graphite addition has low CTE, low density, high thermal conductivity, high melting point and high shock wave damping. The properties are similar to Mo-CD but the mechanical strength is not yet satisfactory. One of the solutions is to use Mo-Gr/Mo sandwich which consists of Mo-Gr core with Mo layers.

HiRadMat at CERN is a novel unique test stand for material testing with proton beams impact. The aims are: to test traditional and novel materials under extreme conditions they may encounter in case of accidental beam impacts; to quantify material damage for LHC operating scenarios; to fully characterize novel materials currently under development for new generation of collimators; to benchmark advanced numerical solutions, in-depth but based on limited and scarce published data on material constitutive models; to collect, mostly in real time, experimental data on constitutive models of materials, including equation of state, strength models, failure models. There are several main objectives for material research and development for accelerator technology. These objectives have been turned into a set of figures of merit (FoM) to assess relevant materials. To reduce RF impedance means to maximize electrical conductivity. To maintain/improve geometrical stability in nominal conditions means to maximize the stability indicator Steady-state Stability Normalized Index (SSNII). To maintain robustness in accidental scenarios means to maximize the robustness indicator Transient Thermal Shock Normalized Index (TSNII); To improve cleaning efficiency by relevant absorption rate means to increase radiation and nuclear interaction length by high enough atomic number; To improve maximum operational temperature means to increase the melting temperature; Additional standard requirements include radiation hardness, UHV (ultra high vacuum) compatibility, industrial producibility of large components, possibility to machine, braze, join, coat, high toughness, acceptable cost

The on-going plans with new material research in the HiRadMat include characterization of six different materials: Intermet180, Glidcop, Molybdenum, Copper-Diamond, Molybdenum-Diamond, Molybdenum-Graphite. There are carried out medium intensity and high intensity tests with different material samples for each material. Each sample holder tier, in the HiRadMat test stand, can host up to 10 specimen. There are 12 tiers. The measurements include: extensive real time data acquisition, in situ and remote, postmortem analyses. The test HiRadMat DAQ equipment includes: remote laser Doppler vibrometer measuring radial velocity of the sample outer cylindrical surface with sampling rate of $5 \mathrm{MHz}$; remote high speed camera which acquires live images of impacted samples with capture rate of $30 \mathrm{kfps}$; in situ strain gauges measuring circumferential and axial strain generated on outer surface; sensors - temperature, vacuum and microphonic. Bringing LHC beyond nominal performances will likely require a new generation of collimators embarking novel advanced materials. Metal matrix composites with diamond reinforcement are particularly appealing as they promise to combine diamond and metal properties.

The vacuum in an accelerator pipe has a dynamic nature. There are a lot of processes accompanying the beam propagation like: beam loss, gas desorption, adsorbed gas at pipe wall, electron loss, capture and charge exchange, multipacting, etc. The collimation system has to avoid avalanching of these processes. Thus, active ion catching methods are used in combination with the collimation. The requirements for a cryocatcher are (L. Bozyk, GSI): desorbed gases must be absorbed again quickly, the deposited energy from lost ions has to be removed, thermal load onto the liquid helium LHe cooling system has to be kept low, activation of surrounding material should be kept low, lost ions should be traced and measured, cryocatcher has to be mounted in a place where the loss distribution is strongly localized, preferably between the quadrupoles. Surrounding cold chamber acts as a cryopump quickly removing desorbed gases, but the cryocatcher temperature has to be kept above the freezing point of the released gases.

\section{Very High Field Magnets}

The aim or work is to construct a HTS (High Temperature Superconductor) dipole insert, generating field of 6T, within a $\mathrm{Nb}_{3} \mathrm{Sn}$ large dipole magnet, generating field 13T. This set of magnets will be used for HI-LHC and HE-LHC machines. The HTS used are Bi-2212 and YBCO coated. The tasks are: specification, characterization and quench modeling; design, construction and test of solenoid insert coils; design, construction and test of dipole insert coils. One of the challenging issues is insert mechanical design, because it will exert round $10^{7} \mathrm{~N} / \mathrm{m}$ pressure onto the surrounding aperture, inside the dipole. The field to be reached is above $20 \mathrm{~T}$.

\section{Muon Ionization Cooling Experiment - MiCE}

MICE (Muon Ionization Cooling) experiment, located at ICTF (Ionization Cooling Test Facility), STFC, Rutherford Appleton Laboratory, UK, is a TA (Transnational Activity) in EuCARD and a facility available for granted visits. MICE is a key step for feasibility of neutrino factory and muon collider. The ICTF comprises a specially developed target and beam line at the ISIS proton synchrotron of $800 \mathrm{MeV}$, and installations to supply RF and liquid hydrogen (LH2) (N. McCubbin, STFC, RAL). The beam line provides muons, protons, pions and electrons at $100 \mathrm{MeV} / \mathrm{c}$ to $400 \mathrm{MeV} / \mathrm{c}$. The target date for full MICE is 2016. The facility is open for research grants within the EuCARD TA [mice.iit.edu].

\section{ACCELERATOR BASED NeUtrino EXPERIMENTS}

Since neutrino oscillations have been demonstrated in 1998 at Super Kamiokande with atmospheric neutrinos, the neutrino masses constitute a new question which has no unique answer in the Standard Model (SM). While all other charged fermions receive 'Dirac' masses, neutrinos are neutral and could also receive 'Majorana' masses which allow a transition between neutrinos and antineutrinos i.e. matter and anti-matter. As a consequence, massive neutrinos could have quite naturally 
'sterile' counterparts, thus, contribute to the solution of several well known observations like: baryon asymmetry of the universe, dark matter and behavior of the early universe, which have no explanation within the SM (A. Blondel, Uni. Geneva). The neutrino mixing matrix consists of three angles and a phase. The unknowns are: mass hierarchies except $m_{1}<m_{2}$, $\operatorname{sign}\left(\Delta m_{32}\right)$, phase $\delta$. Known are mixing angles $\theta_{23}, \theta_{12}, \theta_{13}$, and differential masses $\left|\Delta m_{32}\right|, \Delta m_{21}$. Astroparticle based, rough estimations indicate $m_{1}<1 \mathrm{eV}$. The unknowns are: do neutrinos follow the same mass hierarchy as all other fermions; do neutrinos and antineutrinos oscillate the same (CP violation); do neutrinos have jointly Majorana and Dirac mass terms; do sterile neutrinos exist; what are their masses? Thus, the next neutrino experiments should concentrate on mass hierarchy and CPV (CP violation).

The European neutrino community activities were concentrated around BENE in CARE, NEU2012 in EuCARD, Betabeam in Eurisol, superbeam, betabeam and neutrino factory in EURONU, LAGUNA-LBNO. Future neutrino beams are: EUROnu short baseline CERN to Frejus and long baseline $(2300 \mathrm{~km}) \mathrm{CERN}$ to Pyhasalmi in Finland. Massive neutrinos is a most promising field of research in particle physics today, with large discovery potential with considerable consequences. With the recent observations of a large value of mixing angle $\theta_{13}$, the determination of the neutrino mass hierarchy and the $\mathrm{CP}$ phase are the next steps. These fundamental measurements require and justify dedicated long baseline accelerator based experiment.

\section{FLASH LASER DEVELOPMENT}

Flash laser is a user facility since June 2005 and is continued into 2012. It is also a test bench for the European XFEL and the ILC (International Linear Collider). Typical user operation parameters for the second user period (07-09) were: fundamental wavelength $6.8-40 \mathrm{~nm}$, single pulse energy 10-100uJ, pulse duration 10-70fs, peak power 1-5GW, average power $15 \mathrm{~mW}$, spectral width $1 \%$, peak brilliance around $10^{30}$ photons $/ \mathrm{s} / \mathrm{mrad}^{2} / \mathrm{mm}^{2} / 0.1 \%$ bw. The LLRF system of the TESLA accelerator is under development. The main solution bases on VME platform and now is partially shifted to ATCA and uTCA industrial standard to satisfy the requirements of $0.01 \%$ for amplitude stability and $0.01 \mathrm{deg}$ phase stability, with built in extensive diagnostics.

\section{European X-Ray Free Electron Laser E-XFEL}

The E-XFEL is under construction in DESY Hamburg. The Accelerator Consortium consists of 16 institutes that construct the European XFEL (accelerator and photon systems) by contributing in-kind. The over $3 \mathrm{~km}$ machine consists of an injector, bunch compressor, main linac, collimation, beam distribution, undulators, and experimental photon beams. The $17.5 \mathrm{GeV}$ accelerator complex consists of 100 accelerator modules with 800 TESLA, $1.3 \mathrm{GHz}$ accelerating cavities of around $24 \mathrm{MV} / \mathrm{m}$ field gradient. $25 \mathrm{RF}$ stations, 5.2MW each will provide power. Tight scheduling includes: construction, infrastructure and accelerator component fabrication, start of injector commissioning mid 2014, linac commissioning mid 2015, observe first SASE by end of 2015 (H. Schlarb, DESY).

The E-XFEL parameters set is: electron energy $17.5 \mathrm{GeV}$, bunch charge $1 \mathrm{nC}$, peak current $5 \mathrm{kA}$, slice emittance 0.4-1.0mm mrad, slice energy spread 4-2MeV, shortest SASE wavelength $0.05 \mathrm{~nm}$, pulse repetition rate $10 \mathrm{~Hz}$, bunches per pulse 2700. Worldwide, approximately 3009 cell cavities were produced over the last 15 years. The E-XFEL requires 800 cavities at a production rate of up to 8 cavities per week and 1 module per week. Acceptance testing is a challenge by itself and requires a large infrastructure. The following parts of the project are under realization: niobium cavities, testing infrastructure, cold mass and vacuum vessels, cold magnets and current leads, RF power couplers, infrastructure for string and module assembly, test stand infrastructure, cold linac infrastructure, high power RF system, waveguide distribution, longitudinal beam dynamics control and management, LLRF system, LLRF prototype tests at FLASH, beam operation using uTCA platform, multiple gradient/phase operations, large beam loading with $9 \mathrm{~mA}$ run, beam based feedback, beam diagnostics, beam distribution, undulators, and beam dumps. The following scientific instruments are predicted for the E-XFEL, for hard X-rays: SPB - ultrafast coherent diffraction imaging of single particles, clusters and biomolecules - structure determination of single particles, atomic clusters, bio-molecules, virus particles, cells; MID materials imaging and dynamics - structure determination of nano-devices and dynamics at the nano scale; FXE - femtosecond X-ray experiments - time resolved investigations of the dynamics of solids, liquids and gases; HED - high energy density matter - investigation of matter under extreme conditions using hard X-ray FEL radiation, probing dense plasmas, plasma acceleration of particles. The following experiments are predicted for soft X-rays: SQS - small quantum systems investigation of atoms, ions molecules and clusters in intense fields and non-linear phenomena; SCS - soft X-ray coherent scattering and spectroscopy - electronic and real structure, dynamics of nano-systems and non-reproducible biological objects.

\section{LASER - Plasma Accelerators}

The laser plasma acceleration mechanism is as follows. The rising edge of an intense laser pulse ionizes the gas and creates a plasma. The laser pulse triggers electric fields with the gradient around $100 \mathrm{GeV}$ in its wake. Trapped electrons are accelerated up to $100 \mathrm{MeV}$ to $1 \mathrm{GeV}$ within a few $\mathrm{mm}$ (C. Thaury, CNRS). Main properties of the accelerated beam are: up to $1 \mathrm{GeV}$ of electron energy gained after a few $\mathrm{mm}$, $10-100 \mathrm{pC}$ charge, fs duration of pulse, energy dispersion $\Delta \mathrm{E} / \mathrm{E} 1-10 \%$, transverse size around $1 \mu \mathrm{m}$, divergence around $1 \mathrm{mrad}$. Because of the large divergence, conventional emittance measurements techniques are not suitable. In this acceleration process, the plasma cavity is considered as an undulator and the light generation is based on betatron emission harmonic oscillator in the transverse direction. The principle of colliding injection is to use two beams - a pump beam builds accelerating structures, while an injection beam is for local 
e injection. During the collision, some electrons are heated by the beat wave ponderomotive force (injection phase) and they gain enough energy to be trapped (acceleration phase). The acceleration length is tuned by moving the collision position. Betatron radiation can be efficiently used for emittance measurements in the process of laser plasma acceleration.

\section{ACCELERATOR APPLICATIONS - MYRRHA TRANSMUTATION}

MYRRHA (Multi-purpose hybrid research reactor for hightech applications) is a new large multi-purpose fast neutron research infrastructure, to be located in Mol, Belgium, and operational in 2023. It is an ADS demonstrator (accelerator driven systems). The construction phase of MYRRHA will start in 2015, at the end of the EURATOM FP7 projects like CDT, FREYA and MAX. The aim of these projects is to reach a sufficient level of design for ADS - accelerator driven systems. The ADS accelerator reference scheme is based on a $600 \mathrm{MeV}, 4 \mathrm{~mA} \mathrm{CW}$ superconducting proton linac and up to $100 \mathrm{MW}$ sub-critical reactor of high efficiency, working with highly enriched MOX fuel, $\mathrm{Pb}-\mathrm{Bi}$ eutectic coolant and target. In this architecture MYRRHA is a multipurpose flexible irradiation facility. The research and development is focused on the reliability issues. This may bring substantial impact for availability optimization in future accelerator projects featuring high power proton beams (J-L. Biarrotte, IN2P3, CNRS). MAX - MYRRHA accelerator experiment is a research and development program realized inside EU FP7. ADS are going to be used in the near future in partitioning and transmutation factories. The motivation for this technology is very strong in Europe, especially because most of the European and worldwide reactors are more than 50 years old. About 2500 tons of spent fuel are produced every year by the 145 reactors of EU. In this amount, high level wastes represent $0.2 \%$ in volume and $95 \%$ in radiotoxicity and are long term dominated minor actinides, like Am, Cm (Am-241 in particular). Partitioning and transmutation strategy reduces radiotoxicity and volume of long-lived nuclear wastes before geological storage. The aim is to decrease the radiotoxicity below the level of natural uranium, after around 100 years, which requires volume reduction $100 \mathrm{x}$ and duration reduction 1000x. Transmutation of minor actinides (MA) requires a fast neutron spectrum. The choice of reactor for this process is a sensitive compromise between safety, efficiency, proliferation and politics. The present reference solution for a dedicated transmuter facility is an ADS sub-critical system. MYRRHA is to demonstrate: the physic and technology of an ADS for transmuting long-lived radioactive waste; the ADS concept of infrastructure consisting of coupling accelerator, spallation source and power reactor; practical realization of the transmutation of experimental assemblies. MYRRHA reactor present layout consists of: a vessel, cover, core barrel, core support plate, core plugs, above core structure, heat exchangers, pumps, diaphragm, core and fuel manipulators. Main applications of the MYRRHA irradiation facility are foreseen as follows: test and qualification of innovative fuels and materials for the future generation IV fast, liquid-metal based reactor concepts and for fusion energy; production of radio-isotopes for nuclear medicine, especially ${ }^{99} \mathrm{Mo}$; production of neutron irradiated silicon to enable technologies of renewable energies like windmills, solar panels, electric cars, and fundamental science.

\section{EUCARD 2}

The Enhanced European Coordinated Accelerator Research and Development is a new EU FP7 Integrating Activity initiative for 2013/2017 (M. Vretenar, CERN). EU FP6 CARE (2004-2009) and FP7 EuCARD (2009-2013) projects had an enormous impact in structuring and promoting the European research and development on particle accelerators, but the work needs to be continued. The accelerator community, under the coordination of ESGARD (European Steering Group on Accelerator R\&D), has underlined the importance of continuing a joint R\&D effort and has acknowledged the added value from European projects. The new project has to adapt the new EU guidelines, add new priorities originating from the accelerator field, and to coordinate with other accelerator projects. EuCARD-2 was submitted to EU in November 2011 for a total budget around $30 \mathrm{M}$, with a requested EU contribution 10M. Project was favorably evaluated in March 2012 and the intention is to start it in 2013. The guidelines from EU include: increase the share on Networking Activities, reduce the funding of JRA compensated by increased focus; accent on innovation and outreach to industry, introduction of new networks on innovative technologies, applications and technology transfer; Transnational Accesses remain an essential ingredient of IAs (Integrating Activity); some preference for high-risk, high pay-off activities; coordination with other accelerator projects, in particular TIARA-PP. The priorities from accelerator community are: in the JRA (Joint Research Activities) continuation of core studies - magnets, acceleration, collimation; opening new programs - very-high field magnets, new acceleration concepts, thin films; in the NA (Network Activities) network on catalyzing innovation, extreme beams, low emittance rings; plasma wakefield acceleration; energy efficiency; and accelerator applications.

EuCARD-2 priorities and motivations stretch from basic research to applications, from networking to enriching the ERA (European Research Area). Accelerators based activities are rapidly growing, in full transformation from basic science to applied science, medicine and industry. The period of EuCARD-2 2013-2017 is marked with many strategic facts: the existing accelerators will be pushed to their extreme performance like LHC; new accelerators enter final design and/or construction phase like XFEL, FAIR, ESS, SuperB, MYRRHA; strategic decisions will be taken for the future accelerators like CLIC, ILC, HE-LHC, LHeC and Neutrinos. Individual projects have their own support structure but general accelerator research and development tends to be second priority for large laboratories and is often left to small institutes and laboratories that do not have the critical mass for breakthrough achievements. EuCARD2 and EuCARD aim at joining experience and infrastructures of the major labs with the intellectual potential and creativity of smaller universities 
and institutions on a few research topics of excellence. Thus, the objective is: to propose and develop ideas and technologies for the evolution of the present accelerator infrastructure, to support the final design and construction of the coming generation of facilities, to impact on the choices to be made on the future generation of accelerators.

XBEAM - Extreme Beams is a scientific network dealing with colliders and accelerator frontiers, for high-intensity high-luminosity, including FFAGs (fixed frequency alternating gradient) and superconducting hadron and electron linacs in the interest of HL-LHC, ESS, FAIR, SuperB, HE-LHC, LHeC. LOWeRINGS - Low Emittance Rings is a scientific network seeking for new synergy between synchrotron light sources, storage rings, damping rings and lepton colliders facilities. These subjects are promoted by ICFA (International Committee for Future Accelerators). Energy Efficiency (EEN) is a technological network gathering interest from several laboratories on optimized energy management for a sustainable accelerator science. EE Network wants to be very hands-on and focus on fundamental and precise themes like energy recovery from cooling, efficient klystrons, energy storage, virtual power plant, low-power transport channels. Accelerator Application, a technological network, aims at reviewing and analyzing present applications, propose how to adapt existing accelerator technology to industry, health care, energy and security. Catalyzing Innovation is a technology transfer network based on the existing CERN and STFC structures. It is characterized by proactive approach, scouting for technologies ready for industry engagement and collaboration. EuroNNAc2 is a 'future' network federating the European effort in plasmabased accelerators to prepare a roadmap for an efficient use in full-scale accelerators - from plasma acceleration to plasma based accelerators. Transnational Access will deal with: ion cooling test facility in ICTF of STFC in the UK - tests with high quality low-energy beams; HiRadMat facility at CERN - performance of materials bombarded with intense proton beams; MagNet test stand at CERN - superconducting cable and magnet test station freed after the completion of the LHC.

EuCARD-2 features six JRAs. Future Magnets deals with the development of an HTS (High Temperature Superconductor) magnet that can become the full bore high-filed insert to allow a dipole magnet reaching $20 \mathrm{~T}$ for the needs of a HELHC $2 \times 16.5 \mathrm{TeV}$. The aim is to start with the preliminary step production of the first HTS 10kA high-quality low-loss cable for accelerator SC magnets. Collimators and High Brightness Beams looks for steady increase in LHC luminosity which leads to accelerate the next phase of collimators, validating the new materials and developing the design for the next generation of collimators. Radio Frequency Technologies include: thin film deposition technologies for superconducting cavities, wakefield extraction and monitoring and cavity testing for high-gradient CLIC structures, HOM analysis for XFEL type cavities, new RF photocathodes based on $\mathrm{Pb}$ and on diamond amplifier cathode. Novel Acceleration Techniques involve: laser plasma acceleration, ultra-fast accelerator science and long plasmas. The aim is to develop laser-driven and protondriven plasma-wakefield acceleration, including femtosecond arrival time control.

\section{CONCLUSIONS}

The European research community of accelerator science, including high energy photonics and photon physics is well structured due to common realization of large EC projects. The first project CARE - Coordination of Accelerator R\&D in Europe was realized within the EU FP6 during 20042009. The EU FP7 Project EuCARD, realized in 2009-2013, is an extremely successful undertaking. It engages several hundred European researchers. A successful continuator of the EuCARD will be the EuCARD-2 realized during the period 2013-2017. This paper was prepared using public and Intranet (internal) materials from the EuCARD web page and work packages, invited presentations, highlight talks, and discussions from the third EuCARD Annual Meeting in Warsaw in April 2012. Some fragments of the text were quoted from the EuCARD $3^{\text {rd }}$ AM presentations.

\section{REFERENCES}

[1] EuCARD Web page: cern.ch/eucard and INDICO CERN.

[2] R. S. Romaniuk, "Accelerator science and technology in Europe EuCARD 2012," Proceedings of SPIE, vol. 8454, 2012, art no. 845400 .

[3] — "Accelerator technology and high energy physics experiments, Photonics Applications and Web Engineering, Wilga May 2012," Proceedings of SPIE, vol. 8454, 2012, art no. 845404.

[4] A. Zagozdzinska, K. T. Pozniak, and R. S. Romaniuk, "Heavy stable charged particles search by novel pattern comparator processor," Proceedings of SPIE, vol. 8454, 2012, art. no. 84540P.

[5] R. S. Romaniuk, "Astronomy and space technologies, Photonics Applications and Web Engineering, Wilga May 2012," Proceedings of SPIE, vol. 8454,2012 , art. no. 845403 .

[6] —_, "Photon physics and plasma research, Photonics Applications and Web Engineering, Wilga May 2012," Proceedings of SPIE, vol. 8454, 2012, art. no. 845405 .

[7] — , "Optoelectronics devices, sensors, communication and multimedia, Photonics Applications and Web Engineering, Wilga May 2012," Proceedings of SPIE, vol. 8454, 2012, art. no. 845406.

[8] - "Biomedical, artificial intelligence and DNA computing, Photonics Applications and Web Engineering, Wilga May 2012," Proceedings of SPIE, vol. 8454, 2012, art. no. 845407.

[9] J. Gajda and R. S. Romaniuk, "Laser technology and applications 2012 - a preview," Proceedings of SPIE, vol. 8454, 2012, art. no. 845418

[10] B. Niton, K. T. Pozniak, and R. S. Romaniuk, "A plug-in to Eclipse for VHDL sourc codes: functionalities," Proceedings of SPIE, vol. 8454, 2012, art. no. 84542 C.

[11] R. S. Romaniuk, "Wilga photonics and web engineering, January 2012," Proceedings of SPIE, vol. 8454, 2012, art. no. 845402.

[12] B. Mukherjee, K. T. Pozniak, and R. S. Romaniuk et al., "Application of low-cost Gallium Arsenide light-emitting-diodes as kerma dosemeter and fluence monitor for high-energy neutrons," Radiation Protection Dosimetry, vol. 126, no. 1-4, pp. 256-260, 2007.

[13] R. Romaniuk and K. Pozniak, "Metrological aspects of accelerator technology and high energy physics experiments," Measurement Science and Technology, vol. 18, no. 8, 2008, art. no. E01.

[14] P. Fafara, K. T. Pozniak, and R. S. Romaniuk et al., "FPGA-based implementation of a cavity field controller for FLASH and X-FEL," Measurement Science and Technology, vol. 18, no. 8, pp. 2365-2371, 2008.

[15] A. Burd, K. T. Pozniak, and R. S. Romaniuk et al., "Pi of the sky - allsky, real-time search for fast optical transients," New Astronomy, vol. 10 no. 5, pp. 409-416, 2005.

[16] — " "Pi of the sky' - automated search for fast optical transients over the whole sky," Astronomische Nachrichten, vol. 325, no. 6-8, p. 674 2004.

[17] W. Ackerman et al., "Operation of a free-electron laser from the extreme ultraviolet to the water window," Nature Photonics, vol. 1, no. 6, pp. 336-342, 2007.

[18] T. Czarski, K. T. Pozniak, and R. S. Romaniuk et al., "Superconducting cavity driving with FPGA controller," Nuclear Instruments and Methods in Physics Research A, vol. 568, no. 2, pp. 854-862, 2006. 
[19] - "TESLA cavity modeling and digital implementation in FPGA technology for control system development," Nuclear Instruments and Methods in Physics Research A, vol. 556, no. 2, pp. 565-576, 2006.

[20] —, "Cavity parameters identification for TESLA control system development," Nuclear Instruments and Methods in Physics Research A, vol. 548, no. 3, pp. 283-297, 2005.

[21] R. S. Romaniuk, "Petabit photonic internet," Photonics Letters of Poland, vol. 3, no. 2, pp. 91-93, 2011

[22] P. Obroślak, K. T. Pozniak, and R. S. Romaniuk et al., "Digital techniques for noise reduction in CCD detectors," Photonics Letters of Poland, vol. 2, no. 3, pp. 134-136, 2010.

[23] R. Romaniuk, "Wilga photonics and web engineering 2010," Photonics Letters of Poland, vol. 2, no. 2, pp. 55-57, 2010.

[24] R. Romaniuk and K. T. Pozniak et al., "Optical network and fpga/dsp based control system for free electon laser," Bulletin of the Polish Academy of Sciences: Technical Sciences, vol. 53, no. 2, pp. 123-138, 2005.

[25] G. Kasprowicz and R. S. Romaniuk et al., "CCD detectors for wide field optical astronomy," Photonics Letters of Poland, vol. 1, no. 2, pp. 82-84, 2009.

[26] R. S. Romaniuk, "Wilga symposium on photonics applications," Photonics Letters of Poland, vol. 1, no. 2, pp. 46-48, 2009.

[27] J. R. Just and R. S. Romaniuk, "Highly parallel distributed computing system with optical interconnections," Microprocessing and Microprogramming, vol. 27, no. 1-5, pp. 489-493, 1989

[28] R. Romaniuk, "POLFEL - A free electron laser in Poland," Photonics Letters of Poland, vol. 1, no. 3, pp. 103-105, 2009.

[29] — - "Search for ultimate throughput in ultra-broadband photonic Internet," International Journal of Electronics and Telecommunications, vol. 57 , no. 4 , pp. 523-528, 2011

[30] _ "Photonics and web engineering 2011," International Journal of Electronics and Telecommunications, vol. 57, no. 3, pp. 421-428, 2011.

[31] — "Accelerator infrastructure in Europe EuCARD 2011," International Journal of Electronics and Telecommunications, vol. 57, no. 3 , pp. 413-419, 2011.

[32] — "EuCARD 2010 accelerator technology in Europe," International Journal of Electronics and Telecommunications, vol. 56, no. 4, pp. 485488, 2010.

[33] T. Czarski, K. T. Pozniak, and R. S. Romaniuk, "Control system modeling for superconducting accelerators," in IEEE Instrumentation and Measurement Technology, IEEE Conference Record, 2007, art. no. 4258483

[34] R. Romaniuk, "Advanced photonic and electronic systems Wilga 2010," International Journal of Electronics and Telecommunications, vol. 56, no. 4, pp. 479-484, 2010 .

[35] _ _ "The Photonics Letter of Poland' A new peer-reviewed internet publication of the Photonics Society of Poland," Photonics Letters of Poland, vol. 1, no. 1, pp. 1-3, 2009.

[36] T. R. Wolinski and R. Romaniuk, "Photonics Society of Poland established," Metrology and Measurement Systems, vol. 15, no. 2, pp. 241245, 2008.

[37] S. Chatrchyan et al., "The CMS experiment at the CERN LHC," Journal of Instrumentation, vol. 3, no. 8, 2008, art. no. S08004.

[38] S. Chatrchyan, K. T. Pozniak, R. S. Romaniuk, and W. Zabolotny et al., "The CMS Collaboration, Commissioning of the CMS experiment and the cosmic run at four tesla," Journal of Instrumentation, vol. 5, no. 3, 2010, art. no. T03001.

[39] — "The CMS Collaboration, Performance of the CMS Level-1 trigger during commissioning with cosmic ray muons and LHC beams," Journal of Instrumentation, vol. 5, no. 3, 2010, art. no. T03002.

[40] — "The CMS Collaboration, Performance of the CMS drift-tube chamber local trigger with cosmic rays," Journal of Instrumentation, vol. 5, no. 3, 2010, art. no. T03003.
[41] _ "The CMS Collaboration, Fine synchronization of the CMS muon drift-tube local trigger using cosmic rays," Journal of Instrumentation, vol. 5, no. 3, 2010, art. no. T03004

[42] _ "The CMS Collaboration, Commissioning of the CMS High-Level Trigger with cosmic muons," Journal of Instrumentation, vol. 5, no. 3, 2010, art. no. T03005.

[43] — " "The CMS Collaboration, CMS data processing workflows during an extended cosmic ray run," Journal of Instrumentation, vol. 5, no. 3 , 2010, art. no. T03006.

[44] _ - "The CMS Collaboration, Commissioning and performance of the CMS pixel tracker with cosmic ray muons," Journal of Instrumentation, vol. 5, no. 3, 2010, art. no. T03007.

[45] — " "The CMS Collaboration, Measurement of the muon stopping power in lead tungstate," Journal of Instrumentation, vol. 5, no. 3, 2010, art. no. P03007.

[46] _ - "The CMS Collaboration, Comissioning and performance of the CMS silicon strip tracker with cosmic ray muons," Journal of Instrumentation, vol. 5, no. 3, 2010, art. no. T03008.

[47] — " "The CMS Collaboration, Alignment of the CMS silicon tracker during commissioning with cosmic rays," Journal of Instrumentation, vol. 5, no. 3, 2010, art. no. T03009

[48] _ _ "The CMS Collaboration, Performance and operation of the CMS electromagnetic calorimeter," Journal of Instrumentation, vol. 5, no. 3, 2010, art. no. T03010

[49] — " "The CMS Collaboration, Time reconstruction and performance of the CMS electromagnetic calorimeter," Journal of Instrumentation, vol. 5, no. 3, 2010, art. no. T03011

[50] —, "The CMS Collaboration, Performance of the CMS hadron calorimeter with cosmic ray muons and LHC beam data," Journal of Instrumentation, vol. 5, no. 3, 2010, art. no. T03012.

[51] _ " "The CMS Collaboration, Performance of CMS hadron calorimeter timing and synchronization using test beam, cosmic ray, and LHC beam data," Journal of Instrumentation, vol. 5, no. 3, 2010, art. no. T03013.

[52] — " "The CMS Collaboration, Identification and filtering of uncharacteristic noise in the CMS hadron calorimeter," Journal of Instrumentation, vol. 5, no. 3, 2010, art. no. T03014.

[53] — "The CMS Collaboration, Performance of the CMS drift tube chambers with cosmic rays," Journal of Instrumentation, vol. 5, no. 3 , 2010, art. no. T03015.

[54] — " "The CMS Collaboration, Calibration of the CMS drift tube chambers and measurement of the drift velocity with cosmic rays," Journal of Instrumentation, vol. 5, no. 3, 2010, art. no. T03016.

[55] — "The CMS Collaboration, Performance study of the CMS barrel resistive plate chambers with cosmic rays," Journal of Instrumentation, vol. 5, no. 3, 2010, art. no. T03017.

[56] _ , "The CMS Collaboration, Performance of the CMS cathode strip chambers with cosmic rays," Journal of Instrumentation, vol. 5, no. 3 , 2010, art. no. T03018

[57] _ _ "The CMS Collaboration, Aligning the CMS muon chambers with the muon alignment system during an extended cosmic ray run," Journal of Instrumentation, vol. 5, no. 3, 2010, art. no. T03019.

[58] _ "The CMS Collaboration, Alignment of the CMS muon system with cosmic-ray and beam-halo muons," Journal of Instrumentation, vol. 5, no. 3, 2010, art. no. T03020.

[59] _ _The CMS Collaboration, Precise mapping of the magnetic field in the CMS barrel yoke using cosmic rays," Journal of Instrumentation, vol. 5, no. 3, 2010, art. no. T03021.

[60] _ "The CMS Collaboration, Performance of CMS muon reconstruction in cosmic-ray events," Journal of Instrumentation, vol. 5, no. 3, 2010, art. no. T03022. 